\title{
Correction to: Regeneration of commercial SCR catalyst deactivated by arsenic poisoning in coal-fired power plants
}

\author{
Qiang Lu ${ }^{*, \dagger}$, Zulfiqar Ali*, Hao Tang*, Tahir Iqbal*, Zulqarnain Arain**, Min-shu Cui*, \\ Ding-jia Liu*, Wen-yan $\mathrm{Li}^{*}$, and Yong-ping Yang* \\ *National Engineering Laboratory for Biomass Power Generation Equipment, \\ North China Electric Power University, Beijing 102206, China \\ **Department of Energy System Engineering, Sukkur IBA University, Sukkur, Pakistan \\ (Received 23 October 2018 - accepted 14 January 2019)
}

The article Regeneration of commercial SCR catalyst deactivated by arsenic poisoning in coal-fired power plants, written by Qiang $\mathrm{Lu}^{*, \dagger}$, Zulfiqar Ali*, Hao Tang*, Tahir Iqbal*, Zulqarnain Arain**, Min-shu Cui*, Ding-jia Liu*, Wen-yan Li*, and Yong-ping Yang*, was originally published on the publisher's internet portal (currently
SPringerLink) on 08 February 2019 with misprinted DOI number, 10.1007/s11814-018-0227-9, due to the technical error from converting manuscript file from Microsoft Word to PDF. The correct DOI number for the article is 10.1007/s11814-019-0314-y.

${ }^{\dagger}$ To whom correspondence should be addressed.

E-mail: qianglu@mail.ustc.edu.cn, qlu@ncepu.edu.cn

Copyright by The Korean Institute of Chemical Engineers. 